\title{
Biologia do psilídeo-de-concha Glycaspis brimblecombei Moore (Hemiptera, Psyllidae) em Eucalyptus spp.
}

\author{
Daniela Cristina Firmino-Winckler ${ }^{1}$, Carlos Frederico Wilcken ${ }^{1}$, Nádia Cristina de Oliveira² \\ $\&$ Carlos Alberto Oliveira de Matos ${ }^{3}$
}

\begin{abstract}
1Departamento de Produção Vegetal, Faculdade de Ciências Agronômicas, Universidade Estadual Paulista. Caixa Postal 237, 18603-970 São Paulo-SP, Brasil. dcfirmino@fca.unesp.br; cwilcken@fca.unesp.br

${ }^{2}$ Faculdade Integrado de Campo Mourão - Campus Universitário, Rodovia BR 158 KM 207, s/n, 87300-970 Campo Mourão-PR, Brasil. nadia.oliveira@grupointegrado.br

${ }^{3}$ Campus Experimental de Itapeva, UNESP, Itapeva-SP, Brasil.
\end{abstract}

\begin{abstract}
Red gum lerp psyllid Glycaspis brimblecombei Moore (Hemiptera, Psylidae) biology in Eucalyptus spp.. Glycaspis brimblecombei Moore, also known as red gum lerp psyllid, is characterized to be specific to the genus Eucalyptus. This work aimed to evaluate G. brimblecombei biological cycle in Eucalyptus spp. The work was accomplished in acclimatized chamber (BOD), with temperature of $26{ }^{\circ} \mathrm{C}$ and photophase of 12 hours. Eucalyptus species used in this study were: Eucalyptus camaldulensis, E. tereticornis, E. urophylla, E. grandis, Corymbia citriodora and E grandis x E. urophylla hybrid ('urograndis'). Initially 100 replications (nymphs) per treatment (Eucalyptus species) were prepared. The evaluations were daily and the following biological parameters were measured: nymph phase duration and viability, adult longevity, number of eggs/female, duration of incubation period, egg number and viability, total cycle, longevity and duration of adults. The results demonstrated that, except for C. citriodora, the other eucalypt species offer conditions to G. brimblecombei biological development, being E. camaldulensis the most adequate species.
\end{abstract}

KEYWORDS. Lerp psyllid; pest forest; forest protection.

RESUMO. Biologia do psilídeo-de-concha Glycaspis brimblecombei Moore (Hemiptera, Psyllidae) em Eucalyptus spp.. Glycaspis brimblecombei Moore também conhecido por psilídeo-de-concha, se caracteriza por ser uma espécie específica ao gênero Eucalyptus L'Her. Este trabalho teve por objetivo determinar o ciclo biológico de G. brimblecombei em Eucalyptus spp. O trabalho foi conduzido em câmara climatizada (BOD), sob a temperatura de $26{ }^{\circ} \mathrm{C}$ e fotofase de 12 horas. As espécies de Eucalyptus utilizadas para o experimento foram: Eucalyptus camaldulensis, E. tereticornis, E. urophylla, E. grandis, Corymbia citriodora e um híbrido de E. grandis x E. urophylla ('urograndis'). Inicialmente foram utilizados 100 repetições (ninfas) tratamento (espécies de Eucalyptus). As avaliações foram diárias. Os parâmetros biológicos avaliados foram a duração e viabilidade do estágio ninfal, longevidade dos adultos, número de posturas/fêmea, duração do período embrionário, número e viabilidade ovos, longevidade dos adultos e duração do ciclo total. Os resultados obtidos demonstraram que salvo C. citriodora que apresentou ser letal ao desenvolvimento ninfal de $G$. brimblecombei as demais espécies de Eucalyptus testadas oferecem condições ao desenvolvimento biológico deste psilídeo, sendo que neste trabalho E. camaldulensis mostrou-se a mais adequada.

PALAVRAS-CHAVE. Psilídeo-de-concha; Praga florestal; proteção florestal.

O gênero Eucalyptus (Myrtaceae) é originário da Austrália e foi introduzido no Brasil na segunda metade do Séc. XIX. Atualmente o Brasil tem a segunda maior área plantada de Eucalyptus no mundo e que se destina principalmente à produção de celulose, papel, chapas de fibra e carvão vegetal (Ministério da Ciência e Tecnologia 2004). Os plantios de Eucalyptus se destacam por ocuparem extensas áreas, oferecendo assim maior quantidade de alimento e abrigo para insetos-pragas.

No ano de 2003 foi verificada pela primeira vez no Brasil a ocorrência de Glycaspis brimblecombei Moore (Hemiptera, Psyllidae) também conhecido por psilídeo-de-concha atacando plantios de Eucalyptus spp. primeiramente no estado de São Paulo (Wilcken et al. 2003; Santana \& Burckhardt, 2007). Atualmente, esta espécie encontra-se distribuída também em plantios comerciais de Eucalyptus spp. nos estados de Minas Gerais, Mato Grosso do Sul, Paraná, Goiás (Wilcken et al. 2003; Santana et al. 2003) e Santa Catarina (Lutinski et al. 2006).

Os psilídeos em geral, alimentam-se da seiva das plantas hospedeiras e possuem grande preferência por brotações e folhas novas. O gênero Glycaspis é originário da Austrália e caracteriza-se por se alimentar exclusivamente de espécies do gênero Eucalyptus e por sua infestação ser facilmente reconhecida devido a presença de secreção açucarada e proteção em forma de concha sobre as ninfas (Halbert et al. 2001).

Segundo Tovar et al. (s.d.) as ninfas de G. brimblecombei são achatadas dorso ventralmente com coloração amarela nos três primeiros ínstares. Nos dois últimos ínstares a coloração varia do amarelo ao verde com o abdômen de coloração escura. Os adultos apresentam em geral coloração verde, sendo que as fêmeas podem variar do verde ao vermelho e as antenas são filiformes com 10 artículos. Em geral fêmeas apresentam o tamanho maior que os machos e a parte terminal do abdome arredondado sendo nos machos com projeções chamadas "fórceps".

O ataque de G. brimblecombei causa a descoloração das folhas, indução do aparecimento de fumagina, redução da área 
fotossintética, secamento dos ponteiros e atraso no crescimento das árvores (Wilcken et al. 2003; Carne \& Taylor 1984).

O presente trabalho teve por objetivo determinar o ciclo biológico do psilideo-de-concha G. brimblecombei em diferentes espécies de eucalipto.

\section{MATERIALE MÉTODOS}

O trabalho foi desenvolvido no Laboratório de Controle Biológico de Pragas Florestais do Departamento de Produção Vegetal da Faculdade de Ciências Agronômicas, UNESP Campus de Botucatu.

Para a realização do experimento foram utilizados discos de folhas $(2 \mathrm{~cm}$ de diâmetro) das seguintes espécies de Eucalyptus: E. camaldulensis Dehn, E. grandis Hill Ex Maiden, E. urophylla S. T. Bak, E. tereticornis Smith, o clone de E. grandis x E. urophylla 'urograndis' (VR 3748) e Corymbia citriodora provenientes do Arboreto de Espécies Florestais da FCA/UNESP - Campus de Botucatu.

O experimento foi conduzido em câmara climatizada (tipo BOD) com temperatura de $26^{\circ} \mathrm{C}$ e fotofase de 12 horas.

Os insetos utilizados no decorrer do trabalho foram provenientes de ovos coletados no campo, levados ao laboratório e colocados em placas de petri ( $2 \mathrm{~cm}$ de diâmetro) até a eclosão das ninfas.

O delineamento experimental foi inteiramente casualizado e em todas as etapas do estudo de desenvolvimento biológico, foram feitas avaliações diárias com auxílio de microscópio estereoscópio com aumento de 100 vezes.

Para avaliar o estágio ninfal de G. brimblecombei foram utilizadas 100 repetições (ninfas) por espécie de Eucalyptus. Logo após a eclosão as ninfas foram transferidas individualmente para placas de petri $(2 \mathrm{~cm}$ de diâmetro) contendo os discos de folhas de Eucalyptus dispostos sobre lamina de água de acordo com a metodologia adotada por Santana (2003). Os discos foliares foram trocados a cada dois dias.

Nesta fase foram avaliados os seguintes parâmetros: período de desenvolvimento ninfal (dias), viabilidade, número e duração (dias) dos ínstares, determinado pelo número de artículos da antena de acordo com Sanchez (2003) e também pela a ecdise. Para a observação das ninfas as conchas eram diariamente removidas com auxílio de uma pinça já que logo após estas reiniciavam a formação de uma nova concha para a sua proteção. Os adultos recém emergidos foram sexados e separados em casais. Os casais foram individualizados em placas de petri contendo neste caso, folha de Eucalyptus sp. sobre um disco de papel-filtro umedecido.

Nesta fase foram avaliados os seguintes parâmetros: proporção sexual (macho: fêmea), longevidade, período de oviposição (dias), número de posturas e de ovos por fêmea. Diariamente, após a contagem dos ovos, as posturas obtidas de cada casal/espécie de Eucalyptus foram transferidas para outra placa de petri devidamente identificada, onde permaneceram sob observações até a eclosão das ninfas.

Nesta fase foram avaliados os seguintes parâmetros: período de incubação e viabilidade. Determinou-se o ciclo total (período ninfal + longevidade dos adultos) levando em consideração os dados obtidos para as 100 repetições (indivíduos) para cada espécie de Eucalyptus testados.

Os dados obtidos foram submetidos à análise de variância não paramétrica de Kruskal-Wallis e as médias comparadas pelo teste de Nemenyi $(\mathrm{P}>0,05)$.

\section{RESULTADOSEDISCUSSÃO}

Estágio ninfal. A duração do estágio ninfal de $G$. brimblecombei variou de 12 a 22 dias para os indivíduos mantidos nas diferentes espécies de Eucalyptus. O período médio de desenvolvimento ninfal no híbrido ‘ürograndis' foi de 15,7 dias diferindo significativamente das ninfas mantidas em E. urophylla (14,6 dias) e E. camaldulensis. Em E. grandis não se observou diferença significativa na duração media deste estágio quando comparada com as demais espécies estudadas. Os indivíduos mantidos em E. camaldulensis e E. urophylla não apresentaram entre si diferença significativa na duração do estágio ninfal (Tab. I).

As ninfas de $1^{\circ}, 2^{\circ}, 3^{\circ}, 4^{\circ}$ e $5^{\circ}$ ínstares apresentaram três, cinco, sete e nove artículos antenais, respectivamente. Essas características foram semelhantes às observadas por Sanchez (2003).

A duração média dos ínstares para as ninfas criadas nas diferentes espécies de Eucalyptus foi muito próxima. Observouse que no híbrido 'urograndis' o $1^{\circ}$ instar teve duração média de 4,4 dias sendo que para as demais espécies a duração média foi em torno de 3 dias. As ninfas de $5^{\circ}$ instar apresentaram uma duração média de 3 dias em todas as espécies de Eucalyptus estudadas. As ninfas de $2^{\circ}, 3^{\circ}$ e $4^{\circ}$ instar apresentaram uma duração média de 2,5 dias.

Com relação à viabilidade ninfal, esta foi menor no híbrido 'urograndis' (25\%) e maior em E. camaldulensis (74\%). Em E. grandis, E. urophylla e E. tereticornis a viabilidade ninfal foi de $55 \%, 64 \%$ e $71 \%$, respectivamente. Em C. citriodora a viabilidade foi de $0 \%$ por ter ocorrido uma mortalidade de $100 \%$ das ninfas ainda no primeiro instar.

Estágio adulto. A proporção sexual (macho: fêmea) e razão

Tabela I. Duração em dias (média \pm erro padrão) do estágio ninfal, adulto e ciclo total de Glycaspis brimblecombei mantidos em folhas de diferentes espécies de eucalipto (Temperatura de $26 \pm 1{ }^{\circ} \mathrm{C}$ e fotofase de $12 \mathrm{~h}$ ).

\begin{tabular}{cccc}
\hline Tratamento & $\begin{array}{c}\text { Estágio ninfal } \\
(\mathrm{n}){ }^{1}\end{array}$ & $\begin{array}{c}\text { Estágio adulto } \\
(\mathrm{n}){ }^{1}\end{array}$ & $\begin{array}{c}\text { Ciclo total } \\
(\mathrm{n}){ }^{1}\end{array}$ \\
\hline Híbrido & $15,7 \pm 0,36 \mathrm{ab}$ & $6,0 \pm 0,62 \mathrm{~b}$ & $21,6 \pm 0,62 \mathrm{abc}$ \\
$(\mathrm{n}=25)$ & $(\mathrm{n}=25)$ & $(\mathrm{n}=25)$ \\
'urograndis & $6,3 \pm 0,30 \mathrm{~b}$ & $21,0 \pm 0,40 \mathrm{c}$ \\
E. urophylla & $14,6 \pm 0,30 \mathrm{c}$ & $6,64)$ \\
& $(\mathrm{n}=64)$ & $(\mathrm{n}=64)$ & $(\mathrm{n}=64)$ \\
E. grandis & $15,1 \pm 0,25 \mathrm{abc}$ & $6,2 \pm 0,31 \mathrm{~b}$ & $21,3 \pm 0,39 \mathrm{bc}$ \\
& $(\mathrm{n}=55)$ & $(\mathrm{n}=55)$ & $(\mathrm{n}=55)$ \\
E. camaldulensis & $14,2 \pm 0,16 \mathrm{c}$ & $8,4 \pm 0,39 \mathrm{a}$ & $22,7 \pm 0,41 \mathrm{ab}$ \\
& $(\mathrm{n}=74)$ & $(\mathrm{n}=74)$ & $(\mathrm{n}=74)$ \\
E. tereticornis & $16,0 \pm 0,27 \mathrm{a}$ & $8,0 \pm 0,38 \mathrm{a}$ & $24,0 \pm 0,45 \mathrm{a}$ \\
& $(\mathrm{n}=71)$ & $(\mathrm{n}=71)$ & $(\mathrm{n}=71)$ \\
C. citriodora & - & - & -
\end{tabular}

Médias seguidas das mesmas letras na coluna não diferem entre si pelo teste de Nemenyi $(\mathrm{P} \leq 0,05)$.

${ }^{1}$ Número de indivíduos 
Tabela II. Número de posturas e ovos/fêmea (média \pm erro padrão) e período de incubação em dias (média \pm erro padrão) de ovos de Glycaspis brimblecombei mantidos em folhas de diferentes espécies de eucalipto (Temperatura de $26 \pm 1^{\circ} \mathrm{C}$ e fotofase de $12 \mathrm{~h}$ ).

\begin{tabular}{|c|c|c|c|}
\hline Tratamento & $\begin{array}{l}\text { Posturas/fêmea } \\
\text { (n) }{ }^{1}\end{array}$ & $\begin{array}{l}\text { Ovos/fêmea } \\
\text { (n) }{ }^{1}\end{array}$ & $\begin{array}{c}\text { Incubação } \\
(\mathrm{n})^{2}\end{array}$ \\
\hline $\begin{array}{l}\text { Híbrido } \\
\text { 'urograndis' }\end{array}$ & $\begin{array}{c}2,0 \pm 0,63 \mathrm{a} \\
(\mathrm{n}=5)\end{array}$ & $\begin{array}{c}35,0 \pm 9,54 \mathrm{a} \\
(\mathrm{n}=5)\end{array}$ & $\begin{array}{c}7,5 \pm 0,12 b \\
(n=129)\end{array}$ \\
\hline E. urophylla & $\begin{array}{c}1,3 \pm 0,29 a \\
(n=3)\end{array}$ & $\begin{array}{c}22,3 \pm 13,64 \mathrm{a} \\
(\mathrm{n}=3)\end{array}$ & $\begin{array}{c}7,8 \pm 0,14 b \\
(n=39)\end{array}$ \\
\hline E. grandis & $\begin{array}{c}2,8 \pm 0,95 \mathrm{a} \\
(\mathrm{n}=5)\end{array}$ & $\begin{array}{c}12,2 \pm 5,77 \mathrm{a} \\
(\mathrm{n}=5)\end{array}$ & $\begin{array}{c}8,8 \pm 0,32 \mathrm{a} \\
(\mathrm{n}=28)\end{array}$ \\
\hline E. camaldulensis & $\begin{array}{c}3,2 \pm 0,46 \mathrm{a} \\
(\mathrm{n}=19)\end{array}$ & $\begin{array}{c}31,7 \pm 5,32 \mathrm{a} \\
(\mathrm{n}=19)\end{array}$ & $\begin{array}{c}7,9 \pm 0,04 b \\
(n=452)\end{array}$ \\
\hline E. tereticornis & $\begin{array}{c}2,9 \pm 0,50 \mathrm{a} \\
(\mathrm{n}=20)\end{array}$ & $\begin{array}{c}34,7 \pm 7,15 \mathrm{a} \\
(\mathrm{n}=20)\end{array}$ & $\begin{array}{c}8,5 \pm 0,06 b \\
(n=431)\end{array}$ \\
\hline C. citriodora & - & - & - \\
\hline
\end{tabular}

Médias seguidas das mesmas letras na coluna não diferem entre si pelo teste de Nemenyi $(\mathrm{P} \leq 0,05)$.

${ }^{1}$ Número de indivíduos.

${ }^{2}$ Número de ovos.

sexual dos indivíduos de G. brimblecombei mantidos no híbrido 'urograndis', E. urophylla, E. grandis foi de 1:1,5 e 0,6;1,3:1 e 0,$4 ;$ e $1,4: 1$ e 0,4 , respectivamente. Para os indivíduos mantidos em E. camaldulensis e E. tereticornis a proporção sexual foi de 1,2:1 com razão sexual de 0,4 .

Foi verificado que nas condições estudadas, a longevidade dos adultos foi de, no mínimo, 2 dias e, no máximo de 17 dias para os adultos mantidos nas espécies de Eucalyptus testadas.

Entre E. camaldulensis (8,4 dias) e E. tereticornis (8,0 dias) não houve diferença significativa para a longevidade média dos adultos, porém estas diferiram significativamente das outras espécies estudadas em que os indivíduos obtiveram longevidade média em torno de 6 dias (Tab. I).

Tovar et al. (s.d.) constataram que a longevidade dos adultos de G. brimblecombei mantidos em caixas de criação variou de 9 a 13 dias, resultado superior ao encontrado neste trabalho.

Os resultados referentes ao número de ovos/fêmea e ao número de posturas/fêmea estatisticamente não apresentaram diferença significativa entre as espécies de Eucalyptus testadas, provavelmente devido ao número variado de fêmeas que ovipositaram (Tab. II).

Ciclo total. Nas diferentes espécies de Eucalyptus estudadas, a duração do ciclo total de G. brimblecombei variou de 15 a 34 dias. No híbrido 'urograndis' a duração média do ciclo total dos indivíduos não diferiu significativamente quando comparado com os mantidos nas demais espécies de eucalipto testadas (Tab. I).

Estágio de ovo. Com relação ao período médio de incubação dos ovos de G. brimblecombei, não houve diferenças estatísticas significativas entre as espécies de Eucalyptus (Tab. II), apresentando um período médio inferiores àqueles encontrados por Sanchez (2003) que variou de 11 a 15 dias.

A viabilidade dos ovos de G. brimblecombei foi de $73,7 \%$, $58,2 \%, 75,1 \%$ e apenas de $47,5 \%$ para o híbrido 'urograndis', E. urophylla, E. camaldulensis, E. tereticornis e E. grandis, respectivamente.

Os resultados obtidos neste trabalho demonstraram que as espécies E. camaldulensis, E. urophylla, E. grandis, E.tereticornis e o híbrido 'urograndis' oferecem condições favoráveis ao desenvolvimento biológico de $G$. brimblecombei. No entanto, E. camaldulensis e E. teriticornes foram consideradas as mais adequadas por apresentarem maior sobrevivência ao desenvolvimento desta praga ao contrário de $C$. citriodora que nas condições estudadas apresentou efeito letal impossibilitando o desenvolvimento desta espécie de psilídeo logo no primeiro instar ninfal, podendo esta espécie ser considerada como não hospedeira de G. brimblecombei.

Agradecimentos. A CAPES pela bolsa de mestrado concedida ao primeiro autor.

\section{REFERÊNCIAS}

Carne, P. B.; K. Taylor. 1984. Insect pest. In: Hillis, W. E \& A. G. Brown (ed.). Eucalyptus for wood production. 2 ed. Melbourne: CSIRO, Academic Press, p 155-168.

Halbert, S. E.; R. J. Gill \& J. N. Nisson. 2001. Two Eucalyptus psyllids new to Florida (Homoptera: Psyllidae). Entomology Circular 407: $1-2$.

Lutinski, J. A.; C. J. Lutinski \& F. R. M. Garcia. 2006. Primeiro registro de Glycaspis brimblecombei Moore 1964, (Hemíptera: Psyllidae) em Eucalipto no Estado de Santa Catarina, Brasil. Ciência Rural 36: p. 653-655.

Ministério da Ciência e Tecnologia. Especial Projeto Genolyptus. Disponível em: http://www.mct.gov.br/especial/genolyptus4htm . Acesso em: 05 de abril 2004.

Sánchez, B. S. 2003. Aspectos bionómicos del psilídeo del eucalipto Glycaspis brimbecombei Moore (Homoptera: Psylloidea: Spondyliaspididae). Tesis de maestria. Universidad Autónoma Chapingo. División de ciências Forestales. Chapingo, Texcoco, Edo. de México, 76 p.

Santana, D. L. Q. 2003. Ctenarytaina spatulata Taylor, 1997 (Hemiptera: Psyllidae): morfologia, biologia, dinâmica, resistência e danos em Eucalyptus grandis Hill. Ex Maiden. Tese de Doutorado. Universidade Federal do Paraná. Curitiba, 123p.

Santana, D. L. Q.; A. M. Menezes Jr; H. da Silva; A. F. J. Belloo \& R. M. Favaro. 2003. O psilídeo-de-concha (Glycaspis brimblecombei) em eucalipto. EMBRAPA Comunicado Técnico 105: 1-3.

Santana, D. L. Q. \& D. Burkhardt. 2007. Eucalyptus psyllids in Brazil. Journal of Forest Research 12: 337-344.

Tovar, D. C.; Padilla. A, V. J. A. La conchuela del eucalipto y su parasitóide. Chapingo: Universidad Autónoma. División de Ciencias Forestales, s.d. 72 p.

Wilcken, C. F.; E. B. Couto; C. Orlato; P. J. Ferreira-filho \& D. C. Firmino. 2003. Ocorrência do psilídeo-de-concha (Glycaspis brimblecombei) (Hemiptera: Psyllidae) em florestas de eucalipto no Brasil. Circular Técnica - IPEF 201: 1-11.

Recebido em 07/02/2008; aceito em 17/11/2008 